\title{
Perfil dos atendimentos de um serviço móvel de urgência e emergência de uma universidade federal
}

\author{
Profile of the handles of a mobile service of urgency and emergency of a federal university
}

Perfil de las llamadas de un servicio de emergencia mobile de una universidad federal

Silvana Bastos Cogo ${ }^{1 *}$, Taís Foletto Bevilaqua ${ }^{1}$, Larissa Venturini ${ }^{1}$, Fernanda Luisa Buboltz ${ }^{1}$, Laís Mara Caetano da Silva ${ }^{1}$, Márcio Rossato Badke ${ }^{1}$, Elisabeta Albertina Nietsche ${ }^{1}$, Tierle Kosloski Ramos $^{1}$, Andrei Pompeu Antunes ${ }^{1}$, Graciela Dutra Sehnem¹.

\section{RESUMO}

Objetivo: Caracterizar o perfil dos atendimentos de um serviço móvel de urgência e emergência de uma Universidade Federal do interior do Estado do Rio Grande do Sul. Métodos: Estudo quantitativo, descritivo, retrospectivo, que utilizou-se de dados secundários, coletados de fichas de atendimento no período de maio de 2017 a abril de 2018. Os dados foram digitados em planilha no Excel e analisados por meio do software Statistica 13.3. Resultados: A população atendida foi predominantemente de estudantes do sexo feminino $(63,09 \%)$ com idade entre 21 e 30 anos $(37,30 \%)$. Os atendimentos tiveram como maior causa as situações clínicas e traumáticas. Parcela considerável dos usuários $(91,91 \%)$ recebeu atendimento in loco e foi liberado. Conclusão: Nota-se predomínio de estudantes com faixa etária de 21 a 30 anos, devido o estudo ser de um serviço vinculado a uma universidade. O desfecho foi variado e se associa à gravidade de cada caso. Quando a pessoa atendida não apresentou sinais e sintomas graves ou foram manejados in loco, ocorrendo a liberação sem necessidade de encaminhamento para outro serviço de saúde. Assim, a implantação de um serviço de saúde ambulatorial, para além do serviço móvel, acarretaria em melhoria do atendimento para a população universitária.

Palavras-chave: Emergências, Socorro de urgência, Perfil de saúde, Universidades, Serviços médicos de emergência.

\begin{abstract}
Objective: To characterize the profile of the visits of a mobile service of urgency and emergency of a Federal University of the state of Rio Grande do Sul. Methods: A quantitative study, descriptive, retrospective study, which used secondary data, collected from answer sheets in the period from May 2017 to April 2018. The data were entered in a spreadsheet in Excel and analyzed by means of the software Statistica 13.3. Results: The assisted population was predominantly female students $(63.09 \%)$ with ages between 21 and 30 years $(37.30 \%)$. The handles had as a major cause of the clinical situations and traumatic. Considerable portion of users $(91.91 \%)$ received care in loco and was released. Conclusion: It is noted a predominance of students aged 21 to 30 years, because of the study to be a service that is tied to a university. The outcome was varied and is associated with the severity of each case. When the person answered showed no serious signs and symptoms or were managed in loco, occurring the release without the need for referral to another health servisse. Thus, the deployment of an outpatient health service, in addition to the mobile service, would result in improved care for the university population.
\end{abstract}

Keywords: Emergency, Emergency relief, Health profile, Universities, Emergency medical services.

1Universidade Federal de Santa Maria (UFSM), Santa Maria - RS. *E-mail: silvanabastoscogo@gmail.com

SUBMETIDO EM: 7/2020

ACEITO EM: 8/2020

PUBLICADO EM: 11/2020 


\section{RESUMEN}

Objetivo: Caracterizar el perfil de la atención de un servicio móvil de emergencia y emergencia de una Universidad Federal en el interior del Estado de Rio Grande do Sul. Métodos: Estudio cuantitativo, descriptivo y retrospectivo, que utilizó datos secundarios, recogidos de formularios de servicio de mayo de 2017 a abril de 2018. Los datos se introdujeron en una hoja de cálculo en Excel y se analizaron utilizando el software statistica 13.3. Resultados: La población a la que asistieron era predominantemente alumna $(63,09 \%)$ entre 21 y 30 años $(37,30 \%)$. Las causas más importantes fueron las situaciones clínicas y traumáticas. Porcentaje considerable de usuarios $(91,91 \%)$ recibió atención sobre el terreno y fue puesto en libertad. Conclusión: Hay un predominio de estudiantes de 21 a 30 años, debido a que el estudio procede de un servicio vinculado a una universidad. El resultado fue variado y se asocia con la gravedad de cada caso. Cuando la persona atendida no presentó signos y síntomas graves o fue manejada en el acto, la liberación ocurrió sin la necesidad de ser reecionada a otro servicio de salud. Por lo tanto, la implementación de un servicio de salud ambulatorio, además del servicio móvil, daría lugar a una mejor atención a la población universitaria.

Palabras clave: Emergencias, Ayuda de emergencia, Perfil de salud, Universidades, Servicios médicos de emergência.

\section{INTRODUÇÃO}

Os serviços móveis de urgência e emergência caracterizam-se pelo atendimento a pessoas no âmbito préhospitalar, fornecendo assistência qualificada e adequada às demandas que possam levar ao sofrimento, às sequelas ou mesmo à morte, seja por motivo traumático ou agravo da situação de saúde (BRASIL, 20002).

Nesse sentido, destaca-se que as ações que ocorrem antes da chegada ao ambiente hospitalar, e quando rápidas e eficientes, reduzem complicações na saúde da vítima, e impactam positivamente nas taxas de morbimortalidade (LEFUNDES GAA, et al., 2016).

Assim, o atendimento pré-hospitalar (APH) teve início há mais de 30 anos na América do Norte e Europa, porém, um avanço maior e definitivo em termos de doutrina veio ocorrer na Guerra do Vietnã (1962-1973), quando as autoridades norte-americanas perceberam que a atuação de socorristas nos locais de batalha e nos transportes para hospitais reduzia significativamente a mortalidade e aumentava o tempo de sobrevida dos soldados feridos (CRUZ VERMELHA, 2007). Deste modo, emergências em saúde são aquelas em que o atendimento não pode ser protelado, devendo ser imediato (DE MELO SRA, 2018). Nestes casos, se o atendimento não for hábil e precoce, coloca em risco a vida do paciente.

Já as urgências são situações em que o atendimento pode ser prestado em tempo inferior a duas horas (DE MELO SRA, 2018). As situações não urgentes caracterizam-se como aquelas que podem ser encaminhadas a um pronto atendimento ambulatorial ou atendimento ambulatorial convencional. No entanto, devido à carência de pronto atendimentos ambulatoriais, torna-se rotineira a busca por serviços móveis de urgência e emergência para casos que não são preconizados como alvo desses serviços, resultando na sobrecarga dos mesmos (DAMÁSIO D, et al., 2013).

Valendo-se da importância dos serviços de urgência e emergência, em 2003, o Brasil implantou a Política Nacional de Atenção às Urgências, que representou um avanço na assistência do Sistema Único de Saúde (SUS).

O primeiro componente da política a ser implantado foi o Serviço de Atendimento Móvel de Urgência (SAMU), instituído pela Portaria GM n. 1.864/2003 (BRASIL, 2003). Assim, em 2013, o SAMU 192 estava presente em todos os estados brasileiros, com 175 Centrais de Regulação das Urgências que abrangiam em torno de 1955 municípios ou 65\% da população brasileira (BRASIL, 2013).

O Ministério da Saúde estimou que o SAMU alcançaria a cobertura de $100 \%$ da população brasileira em 2018. Machado, Salvador e O'Dwyer (2011), a o alisarem o SAMU como política pública, relatam que, apesar de inspirado nas experiências internacionais, o modelo brasileiro do SAMU adquiriu características próprias, tendo que ser adaptado, de acordo com as condições do país. 
Atualmente, a Portaria $n^{\circ} 1.600$, de 7 de julho de 2011, institui a Rede de Atenção às Urgências e Emergências (RUE) no SUS, que tem como finalidade articular e integrar todos os equipamentos de saúde, tendo como objetivo a ampliação e qualificação do acesso humanizado e integral aos usuários do SUS que se encontram em situação de emergência nos serviços de saúde, de forma ágil e oportuna. Ainda a portaria considera que nos fluxos assistenciais da RUE é necessário o acolhimento com classificação de risco, qualidade e resolutividade em todos os pontos de atenção da rede (BRASIL, 2011).

Assim, preconiza-se, por intermédio desse serviço, disponibilizar atendimento à vítima nos minutos iniciais em que sofreu um agravo à sua saúde, o qual pode levá-la à deficiência física ou à morte, sendo, portanto, imprescindível que forneça atendimento de qualidade e encaminhe a uma porta de entrada adequada ao sistema de saúde, objetivando, assim, a manutenção da vida e a minimização das sequelas (DANTAS RAN, et al., 2015). Entretanto, a extensão de algumas cidades e aspectos de mobilidade urbana pode dificultar o rápido deslocamento de uma equipe de atendimento pré-hospitalar até o local do agravo.

Segundo Ciconet (2015), o tempo resposta de atendimentos de emergência clínicos e traumáticos, como síndromes neurológicas, cardiológicas, dispneias, acidentes de trânsito e quedas, é um importante indicador associado à sobrevivência de pacientes. Assim, a assistência de uma demanda de atenção médica urgente no local onde acontece é um dos maiores avanços na área de urgências. Nesse sentido, o indicador de tempo resposta serve para controlar a qualidade da atenção nos serviços de urgências pré-hospitalares (VILLAVIVENCIO ML, 2008).

Logo, precisa-se considerar que locais com grande circulação de pessoas possuem maior probabilidade na ocorrência de situações com agravantes de saúde. Nessa perspectiva, destacam-se as Universidades Públicas brasileiras que contemplam em seus campi a circulação de milhares de pessoas (discentes, docentes, servidores, moradores de casas universitárias e comunidade em geral).

Nesse ínterim, uma Universidade Pública Federal do interior do Rio Grande do Sul (RS) observou a necessidade da criação de um serviço de atendimento móvel pré-hospitalar, exclusivo para a instituição. A decisão para implementação do serviço ancorou-se a acontecimentos pontuais de emergência, como vítimas de parada cardiorrespiratória (PCR), e outras situações clínicas graves, bem como ao fato da Universidade em questão estar localizada a uma distância considerável de bases de atendimento do SAMU, e de possuir uma grande extensão territorial.

Destaca-se, que essa Universidade é a maior em extensão territorial existente no Brasil, atingindo 2000 hectares, nos quais as edificações perfazem 309.332,72 metros quadrados de área construída no Campus sede. Ainda, possui a casa de moradia estudantil mais populosa da América Latina, com mais de duas mil pessoas vivendo em suas instalações (UNIVERSIDADE FEDERAL DE SANTA MARIA, 2018).

O serviço móvel de urgência e emergência desta universidade iniciou suas atividades em maio de 2017, contando com duas equipes, cada uma composta por médico, enfermeiro, técnico de enfermagem e motorista. Os profissionais foram capacitados e qualificados com cursos de Atendimento Pré-Hospitalar (APH) e regulam os atendimentos por meio de chamado telefônico, deslocando a ambulância com a equipe até o local solicitado, dentro do território da universidade (UNIVERSIDADE FEDERAL DE SANTA MARIA, 2018).

Portanto, reconhece-se a incipiência desta modalidade de serviço dentro do ambiente universitário, o que o torna uma lacuna na literatura corrente. Logo, o presente estudo objetiva caracterizar o perfil dos atendimentos de um serviço móvel de urgência e emergência de uma Universidade federal do interior do Estado do RS.

\section{MÉTODOS}

Estudo quantitativo, descritivo e retrospectivo que teve como cenário em uma Universidade Federal do interior do RS. Em meio a precariedade de serviços de saúde que atendam a demanda da comunidade universitária, esta instituição implementou no ano de 2017 um Serviço Móvel de Urgência e Emergência, que conta com uma equipe especializada em atendimento pré-hospitalar. O serviço está disponível para atender chamados dentro do campus sede desta Universidade, podendo ser acionado por qualquer pessoa que esteja 
circulando pelo local. Funciona durante 12 horas diárias, de segunda a sexta-feira, com duas equipes, divididas em turnos de seis horas, das $7 \mathrm{~h}$ às $13 \mathrm{~h}$ e das $13 \mathrm{~h}$ às $19 \mathrm{~h}$. Cada equipe é formada por um médico, um enfermeiro, um técnico de enfermagem e um motorista.

Este serviço está vinculado à Rede de Saúde do município, podendo referenciar os atendimentos de acordo com a necessidade do usuário. A coleta de dados ocorreu entre março a maio de 2018, constituíramse como fonte as fichas referentes aos atendimentos dos usuários do serviço móvel de urgência e emergência, ocorridos no período de 02 de maio de 2017 a 30 de abril de 2018, totalizando os 12 meses iniciais de seu exercício, totalizando 940 fichas. Foram incluídas as fichas de atendimento com dados preenchidos de maneira adequada, ou seja, com todos os itens preenchidos e com letra legível.

Visando atingir os objetivos propostos, foram selecionadas as seguintes variáveis para categorização: dados demográficos (sexo e faixa etária), atuação (discente, docente, morador da casa estudantil universitária (CEU), técnico administrativo em educação (TAE), trabalhador terceirizado, outro), causa pela procura de atendimento (neurológica, cardiovascular, respiratória, metabólica, traumática, obstétrica e psiquiátrica), bem como o local de destino do encaminhamento.

Tal coleta de dados abarcou todas as fichas de atendimento do serviço móvel de urgência e emergência e, por ter sido um estudo que teve como participantes a população de atendidos, não foi necessária a realização de cálculo amostral. As variáveis categóricas ou qualitativas foram descritas por meio de frequência absoluta e frequência relativa, enquanto as variáveis quantitativas foram analisadas por medidas de posição (média e mediana) e dispersão (desvio padrão). As variáveis quantitativas contínuas foram submetidas ao teste de normalidade de Shapiro-Wilk, o qual identificou a distribuição normal dos dados coletados. Após tal confirmação, procedeu-se o tratamento estatístico. Os dados foram digitados em planilha no Excel e analisados por meio do software Statistica 13.3.

O estudo seguiu os preceitos éticos descritos na resolução no 466/ 2012 do Conselho Nacional de Saúde (BRASIL, 2012) e foi aprovado pelo Comitê de Ética em Pesquisa da Universidade Federal de Santa Maria (UFSM) sob no 2.541.788.

\section{RESULTADOS}

Foram realizados 940 atendimentos pelo serviço no período supracitado, sendo que $63,09 \%$ (593) eram do sexo feminino e $36,91 \%$ (347) do sexo masculino. No Gráfico 1 encontram-se as faixas etárias dos usuários atendidos no serviço móvel de urgência e emergência. Destacaram na procura pelo atendimento os que apresentavam idade entre 21 e 30 anos, correspondendo a 37,3\% (349), seguido pela faixa etária dos 11 aos 20 anos, $27,34 \%$ (257). A média de idade de pessoas atendidas foi de 29 anos e os mais atendidos tinham 21 anos. Dentre os 940 atendimentos analisadas, 27 fichas não continham a idade dos pacientes.

Gráfico 1 - Faixas etárias dos usuários atendidos no Serviço Móvel de Urgência e Emergência.

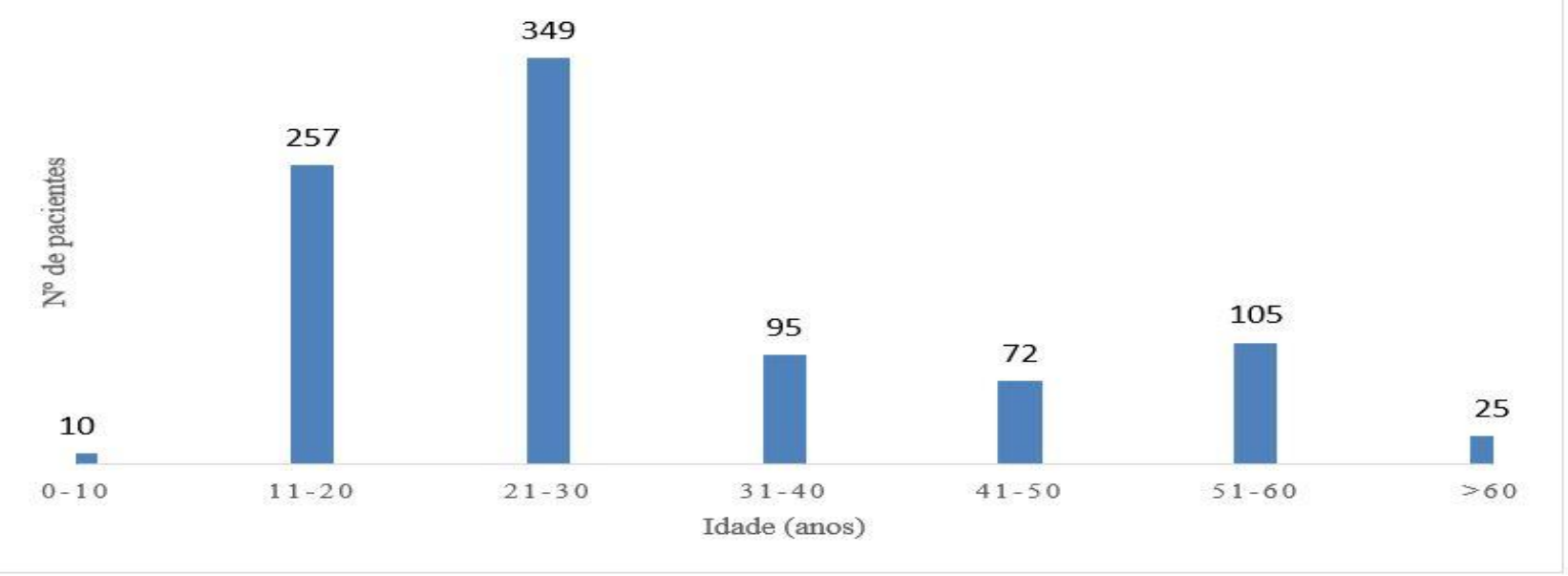

Fonte: BEVILAQUA TF, et al., 2020. 
Quanto à categoria em que cada usuário pertencia, a classificação foi realizada de acordo com sua função ou atividade dentro da universidade, bem como os que não possuíam vínculo com a instituição, estão denominados no Gráfico 2 como outros. Assim, conforme ilustrado no Gráfico 2, estão contempladas 936 fichas de atendimento, e excluídas quatro, por não estarem com este campo preenchido.

Gráfico 2 - Função ou atividade de cada usuário do serviço dentro da universidade.

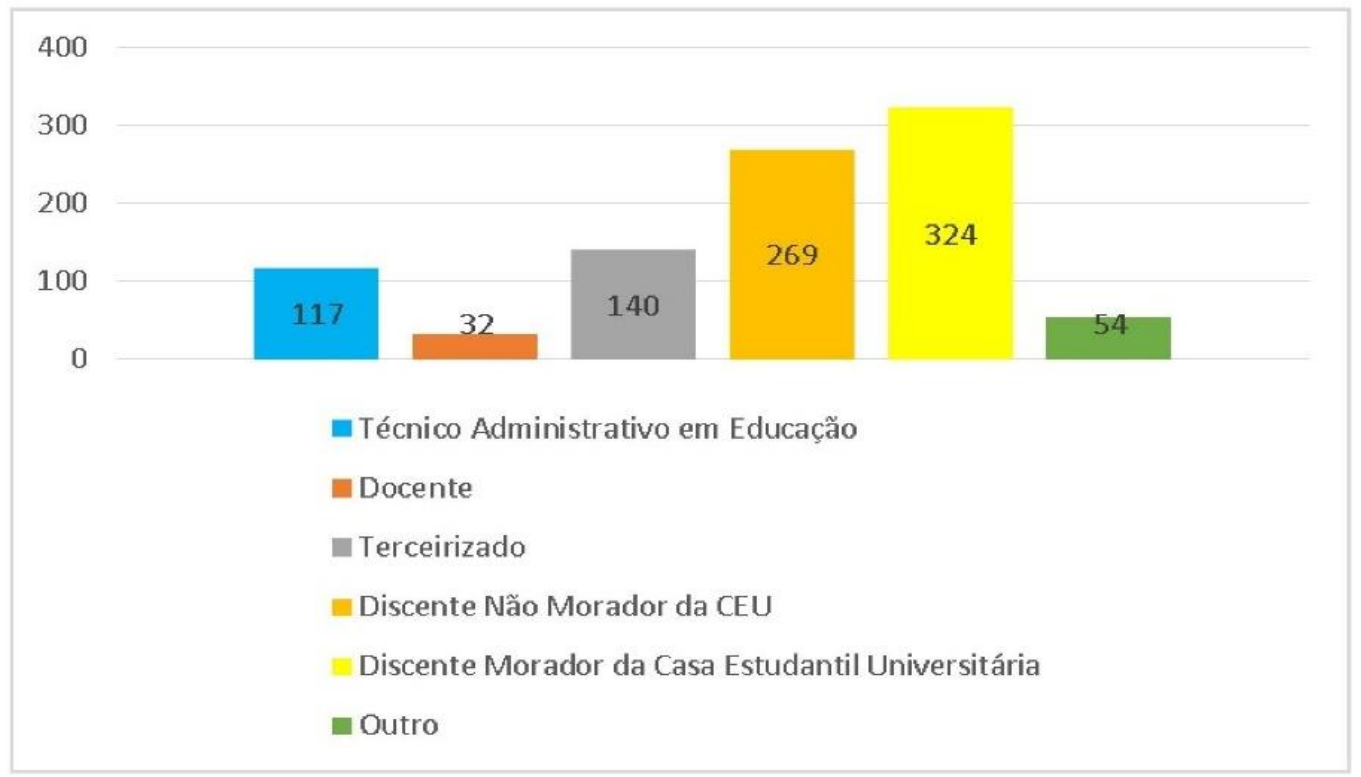

Fonte: BEVILAQUA TF, et al., 2020.

Dentre as causas que motivaram os usuários a procurar pelo atendimento no serviço móvel de urgência e emergência, destacaram-se as lesões traumáticas, responsáveis por 18,19\% (171), conforme apresentados na Tabela 1. Ressalta-se que a motivação descrita como outros, contemplam a otite externa e interna, alterações oculares, problemas relacionados ao trato urinário, dermatites, entre outros. Oito fichas de atendimento não apresentaram o devido preenchimento neste campo, portanto foram excluídas.

Tabela 1 - Causas de agravamento de saúde de usuários atendidos no serviço móvel de urgência e emergência. Santa Maria, RS, Brasil, 2018.

\begin{tabular}{cc} 
Causa & $\mathbf{n}(\%)$ \\
\hline Traumática & $171(18,2)$ \\
Respiratória & $130(14,7)$ \\
Gastrintestinal & $109(11,6)$ \\
Infecciosa & $107(11,3)$ \\
Neurológica & $86(9,1)$ \\
Cardiovascular & $83(8,9)$ \\
Psiquiátrica & $78(8,3)$ \\
Intoxicação & $27(2,9)$ \\
Gineco/obstétrica & $13(1,4)$ \\
Metabólica & $05(0,5)$ \\
Outras & $123(13,1)$ \\
\hline
\end{tabular}

Fonte: BEVILAQUA TF, et al., 2020.

No desfecho do atendimento, a maioria dos usuários atendidos 864 (91,91\%) foram liberados para retornar as suas atividades, enquanto $68(7,23 \%)$ foram encaminhados para unidades hospitalares do mesmo município ou pronto atendimento. Quando houve outro encaminhamento, como para a Atenção Básica, ou unidade hospitalar de outro município, foi categorizado como outro, conforme apresentado na Tabela 2. 
Tabela 2 - Desfecho dos usuários que foram atendidos no serviço móvel de urgência e emergência. Santa Maria, RS, Brasil, 2018.

\begin{tabular}{cc}
\hline Desfecho & $\mathbf{n}(\%)$ \\
\hline Liberado com orientações & $864(92,0)$ \\
Unidades de Pronto-Atendimento & $34(3,6)$ \\
Hospital Público & $17(1,8)$ \\
Hospital Privado & $17(1,8)$ \\
Outro & $08(0,8)$ \\
Total & $940(100)$ \\
\hline
\end{tabular}

Fonte: BEVILAQUA TF, et al., 2020.

O desfecho foi variado e se associa à gravidade de cada caso. Desse modo, quando a pessoa atendida não apresentou sinais e sintomas graves, ou foram manejados in loco, ocorreu a liberação sem necessidade de encaminhamento para outro serviço de saúde. Entretanto, quando foi necessária uma investigação minuciosa, seja por meios clínicos ou laboratoriais, ou manutenção de sua estabilidade hemodinâmica, realizaram-se encaminhamentos a pronto atendimentos ou hospitais, segundo a complexidade e a aceitação do local para onde foi referenciado.

\section{DISCUSSÃO}

O serviço móvel de urgência e emergência que foi cenário deste estudo apresenta semelhança com o modelo de atendimento do SAMU e caracteriza-se como um serviço pioneiro no Brasil. Isso, porque os atendimentos ocorrem exclusivamente dentro do território de uma Universidade.

O perfil dos usuários foi composto por mulheres, que corresponderam à maior parte da demanda atendida (63,09\%). Tal característica é observada em serviços de urgência com diferentes complexidades, visto que resultados semelhantes já foram observados em estudos realizados em outros locais, como em um prontoatendimento do Estado do RS, no qual 53,7\% eram do sexo feminino (OLIVEIRA GN, et al., 2011). No entanto, precisa-se contrapor com o fato de que alguns estudos apresentam a predominância do sexo masculino, principalmente serviços de urgência e emergência pré-hospitalares, o que tem relação com maior exposição masculina à violência urbana.

Estudo realizado em Novo Hamburgo, interior do RS, no contexto pré-hospitalar, identificou que $61,94 \%$ dos atendimentos eram feitos para o sexo masculino (SILVA AMA e SHAMA SFM, 2017), o que demonstra que podem ocorrer variações segundo o contexto do serviço. Pelo fato de estar inserido em uma Universidade, e os casos atendidos não terem sido, em sua maioria, de alta complexidade, fato mensurado pelo desfecho dos atendimentos, observa-se relação com o contexto de pronto-atendimento.

Quanto à idade, a maior procura pelos atendimentos envolveu pessoas com idade entre 21 e 30 anos $(37,30 \%)$. Segundo a pesquisa do perfil sociodemográfico das Instituições Federais de Ensino Superior Brasileiras, em 2014, 70,3\% dos estudantes tinha idade entre 19 e 29 anos. Isso vai ao encontro e justifica o grande percentual de pessoas atendidas nessa faixa etária no serviço de urgência e emergência em estudo, apesar de os atendimentos não serem exclusivos para os discentes, entretanto, por ser demograficamente restrito ao campus universitário, encontram-se mais pessoas dessa faixa etária (ANDIFES, 2016).

Constatou-se também que a maior parte das pessoas atendidas eram discentes, moradores da CEU $(34,46 \%)$ e não moradores $(28,61 \%)$, totalizando $63 \%$ do total dos atendimentos, seguido pelos TAEs $(12,44 \%)$. Tal dado mostra-se em consonância ao quantitativo das categorias que compõe a comunidade universitária, composta por estudantes, docentes e técnicos administrativos (UNIVERSIDADE FEDERAL DE SANTA MARIA, 2018).

Há que se destacar o quantitativo de discentes moradores da CEU que buscaram atendimento, permitindo ênfase ao fato de que esses pertencem a uma das parcelas vulneráveis da instituição, seja pelo perfil econômico ou social. Existem duas Unidades Básicas de Saúde (UBS) que atendem essa demanda, 
pertencentes à Atenção Primária em Saúde (APS), mas devido sua localização estar há aproximadamente quatro quilômetros de distância do campus e ao fato de atenderem sob agendamento, há uma dificuldade de acesso dos discentes à rede de atenção à saúde. Tal dificuldade também pode se dar pelo fato da maioria dos estudantes serem oriundos de outras cidades, estados ou países, o que impacta negativamente no vínculo estabelecido com a APS.

Nota-se também que, por vezes, as queixas das pessoas atendidas nesse serviço são difusas e, talvez, indevidas a este nível assistencial. Desse modo, levando a identificar que estas poderiam ser resolvidas em outros setores, como por exemplo, na APS.

Entretanto, pressupõe-se que, em razão da localização, das mazelas reconhecidas para o acesso aos serviços públicos de saúde e, consequentemente, por este serviço acolher as demandas de atendimento que Ihes são solicitadas, estes fatos culminam para que o serviço seja a primeira escolha das pessoas que estão na universidade, em especial dos estudantes moradores da CEU.

Destaca-se o fato deste serviço atender uma área restrita, ou seja, o campus de uma universidade, e assim contemplar pessoas que se encontram em uma faixa etária jovem, o que pode diminuir a probabilidade de ocorrência de casos graves, explicando a grande maioria de casos clínicos.

Outra parcela significativa foi a dos trabalhadores terceirizados, que totalizou $140(14,89 \%)$ atendimentos. Isso pode estar associado ao fato dos trabalhadores encontrarem dificuldades em acessar outro tipo de atenção à saúde, seja a privada por questão financeira, ou a pública, pela demora em função do agendamento, modelo este funcionante nas UBS próximas do campus, ou pela impossibilidade de se afastar de suas atividades laborais.

Em um estudo realizado na APS em uma grande cidade do estado de São Paulo, observou-se esta mesma impressão por parte dos entrevistados, que descreveram o acesso ao serviço da APS como algo burocrático e demorado, o que vai desde o tempo de espera do agendamento até o dia da consulta, como também o próprio processo de atendimento no serviço (seja este agendado ou não) (CAMPOS RTO, et al., 2014).

Quanto à classificação dos atendimentos, as causas clínicas extrapolam as traumáticas, quando somadas em um grande grupo $(47,02 \%)$, correspondendo à maioria dos registros, precedidas por traumas $(18,19 \%)$. Estudos semelhantes corroboram com a predominância clínica, como foi o caso de uma pesquisa desenvolvida no interior do RS, com predominância de causas clínicas, e causas traumáticas ocupando o segundo lugar. Estas incluem acidentes de trânsito, violência e quedas (CASAGRANDE D, et al., 2013).

No entanto, um estudo coreano demonstra que nos últimos anos, foram alcançadas melhorias significativas na sobrevida após o trauma. Uma das razões para esse progresso foram às melhorias nos serviços médicos de emergência e o transporte de pacientes traumatizados, que salvam vidas, para um centro capaz de fornecer cuidados definitivos.

O transporte rápido e oportuno de pacientes traumatizados para uma instituição de trauma adequada, ou seja, "levar o paciente certo ao lugar certo, no momento certo", é importante para aumentar a taxa de sobrevida em pacientes traumatizados (KIM OH, et al., 2017).

O desfecho mais observado nas fichas de atendimento foi à liberação para o retorno de suas atividades ou para o seu domicílio $(91,91 \%)$, seguido por encaminhamentos para pronto atendimentos municipais $(3,61 \%)$. Reduzida parcela foi encaminhada para a atenção hospitalar (3,59\%), somando hospitais públicos e privados, para aqueles que possuíam plano de saúde. Desfecho semelhante foi encontrado em estudo realizado no serviço de pronto atendimento de um hospital universitário localizado na capital paulista, de alta complexidade, em que são atendidos diariamente cerca de mil pessoas, onde $69,1 \%$ dos pacientes foram liberados após o atendimento (FRANGO BCTM, et al., 2018).

Assim, os acadêmicos ou trabalhadores que recebem o atendimento dentro do campus universitário, a depender da complexidade do seu caso e com a resolutividade do serviço, conseguem retornar a suas atividades, o que sugere precedentes positivos para a instituição, devido a manutenção das atividades acadêmicas e profissionais. 


\section{CONCLUSÃO}

A abordagem do perfil dos atendimentos do serviço móvel de urgência e emergência, localizado em uma Universidade, permitiu apreender a tipologia dos atendimentos, delineamento dos casos e a complexidade dos fatores associados ao ambiente universitário, permeado por um conjunto de circunstâncias que ratificam a necessidade deste serviço de saúde. Os resultados sugerem sobre a possibilidade da importância da criação de um serviço de saúde ambulatorial, para a melhoria do atendimento. Assim, acredita-se que traçar o perfil dos atendimentos do serviço móvel de urgência e emergência contribuiu para aprimorar o serviço de saúde, em especial, à equipe de enfermagem que é a protagonista no que se refere a ações de prevenção, promoção, recuperação e habilitação da saúde, bem como subsidiar os gestores para aprimorar e articular novas políticas de saúde para melhor atender a comunidade universitária.

\section{REFERÊNCIAS}

1. ASSOCIAÇÃO NACIONAL DOS DIRIGENTES DAS INSTITUIÇÕES FEDERAIS DE ENSINO SUPERIOR FÓRUM NACIONAL DE PRÓ-REITORES DE ASSUNTOS COMUNITÁRIOS E ESTUDANTIS (ANDIFES). IV pesquisa do perfil socioeconômico e cultural dos estudantes de graduação das instituições federais de ensino superior brasileiras. Uberlândia, 2016.

2. BRASIL. Ministério da Saúde. Portaria GM/MS n.2048, de 5 de novembro de 2002. Aprova o Regulamento Técnico dos Sistemas Estaduais de Urgência e Emergência. Diário Oficial da União, Brasília: MS, 2002.

3. BRASIL. Ministério da Saúde. Portaria no 1600, de 7 julho de 2011. Reformula a Política Nacional de Atenção às Urgências e institui a Rede de Atenção às Urgências no Sistema Único de Saúde (SUS). Brasília: MS, 2011.

4. BRASIL. Ministério da Saúde. Portaria oํ 1863, de 29 de setembro de 2003. Brasília: MS, 2003.

5. BRASIL. Ministério da Saúde. Resolução n. 466, de 12 de dezembro de 2012. Brasília: MS, 2012.

6. BRASIL. Ministério da Saúde. Sala de Apoio à Gestão Estratégica (SAGE). Brasília: MS, 2013.

7. CAMPOS RTO, et al. Avaliação da qualidade do acesso na atenção primária de uma grande cidade brasileira na perspectiva dos usuários. Revista Saúde Debate, 2014; 38(spe):252-64.

8. CASAGRANDE D, et al. Perfil dos atendimentos realizados por uma Unidade de Suporte Avançado do Serviço de Atendimento Móvel de Urgência (SAMU) do Rio Grande do Sul. Sci med (Porto Alegre, Online), 2013; 23(3):149-55.

9. CICONET RM. Tempo resposta de um serviço de atendimento móvel de urgência. Tese (Doutorado em Enfermagem) - Universidade Federal do Rio Grande do Sul, Escola de Enfermagem, Porto Alegre, RS, 2015.

10. CRUZ VERMELHA BRASILEIRA. Filial do Estado de Minas Gerais. Manual de socorro básico de emergência. 1. ed, 2007; 160p.

11. DAMÁSIO D, et al. Demanda de um Pronto Atendimento 24 horas no Sul de Santa Catarina. Revista Iniciação Científica, 2013; 11(1):118-29.

12. DANTAS RAN, et al. Instrumento para avaliação da qualidade da assistência pré-hospitalar móvel de urgência: validação de conteúdo. Rev Esc Enferm USP, 2015; 49(3):381-7.

13. DE MELO SRA. Caracterização e avaliação dos usuários frequentes (hiperutilizadores) do Serviço público de urgência/emergência do município de Monte Claro/SP. Dissertação (Mestrado em Gestão em Saúde) - Universidade de São Paulo. Faculdade de Medicina de Ribeirão Preto, SP, 2018.

14. FRANGO BCTM, et al. Associação do perfil de usuários frequentes com as características de utilização de um serviço de emergência. REME rev min enferm, 2018; 22:e-1071.

15. KIM OH, et al. Mortalidade Reduzida em Pacientes Gravemente Feridos Utilizando Serviços Médicos de Emergência em Helicópteros Hospitalares no Transporte Inter-Hospitalar. J Korean Med. Sci, 2017; 32 (7): 1187-1194.

16. LEFUNDES GAA, et al. Caracterização das ocorrências do serviço de atendimento móvel de urgência. Rev baiana enferm, 2016; 30(3):1-10.

17. MACHADO CV, et al. Serviço de Atendimento Móvel de Urgência: análise da política brasileira. Revista de Saúde Pública, São Paulo, 2011; v. 45, n. 3.

18. OLIVEIRA GN, et al. Perfil da população atendida em uma unidade de emergência referenciada. Rev latinoam enferm (Online), 2011; 19(3):548-56.

19. SILVA AMA, SHAMA SFM. Epidemiologia do trauma em atendimentos do SAMU Novo Hamburgo/RS no primeiro trimestre de 2015. Revista Saúde e Pesquisa, 2017;10(3):539-48.

20. UNIVERSIDADE FEDERAL DE SANTA MARIA (UFSM). UFSM em números. Santa Maria, 2018.

21. VILLAVICENCIO ML. Tiempo de respuesta em el transporte primário de prioridades I y II en el servicio de sistema de transporte asistido de emergencia STAE-ESSALUD. Emergencias, Madrid, 2008; v. 20, p. 316-321. 